\title{
Sleep's Role in the Consolidation of Emotional Episodic Memories
}

\author{
Jessica D. Payne' and Elizabeth A. Kensinger ${ }^{2}$ \\ 'University of Notre Dame and and ${ }^{2}$ Boston College
}

\begin{abstract}
Emotion has a lasting effect on memory, encouraging certain aspects of our experiences to become durable parts of our memory stores. Although emotion exerts its influence at every phase of memory, this review focuses on emotion's role in the consolidation and transformation of memories over time. Sleep provides ideal conditions for memory consolidation, and recent research demonstrates that manipulating sleep can shed light on the storage and evolution of emotional memories. We provide evidence that sleep enhances the likelihood that select pieces of an experience are stabilized in memory, leading memory for emotional experiences to home in on the aspects of the experience that are most closely tied to the affective response.
\end{abstract}

\section{Keywords}

affect, amygdala, consolidation, emotion, memory, fMRI, hippocampus, sleep, stabilization, trade-off

Episodic memory refers to the long-term retention of contextually rich representations of past experiences. When we remember specific events from our past, recalling their spatial and temporal context or recollecting perceptual or conceptual details of those events, our memory is considered to be episodic in nature. Although our experience of episodic memory occurs at the moment of retrieval, when information about the initial experience enters conscious awareness, successful retrieval is contingent upon the successful completion of at least two earlier stages of memory formation. First, the experience must be properly encoded. Encoding involves transforming new information into a representation capable of being stored in memory, just as the key presses we make on keyboard must be converted to a format that can be stored in a computer. Second, the information must be durably stored in a manner that can withstand the passage of time, a process known as memory consolidation. Only if these processes occur successfully will it be possible for information from the initial event to be retrieved later in time.

The events we care most about remembering are often accompanied by an emotional response, including cognitive, physiological, and somatic reactions. The examination of memory for these experiences - the study of "emotional memory" - has made clear that emotion can intervene at each of the memory phases noted above. Emotion has a powerful influence on memories. It can alter the likelihood that an experience will be encoded in memory, influence how memories are
Science

19(5) 290-295

(C) The Author(s) 2010

Reprints and permission:

sagepub.com/journalsPermissions.nav DOI: 10.1 177/0963721410383978

http://cdps.sagepub.com

@SAGE consolidated, and affect how events are re-experienced at retrieval (see Kensinger, 2009 and McGaugh, 2004 for reviews). Although emotion's influence during each of these phases is critically important, this review focuses on emotion's effect on memory consolidation.

\section{Emotional Memory Consolidation: A Brief Introduction}

A long-standing question in cognitive neuroscience is how memory for emotional experience is consolidated over time. This issue is key to understanding basic mechanisms giving rise to long-lasting emotional memories and for appreciating how those mechanisms may be altered in affective disorders. The occurrence of an emotional reaction enhances the likelihood that an experience will become consolidated. When an emotional reaction occurs, stress hormones (e.g., cortisol, norepinephrine) are released, which in turn leads to increased activation of the amygdala, an almond-shaped region situated

\section{Corresponding Author:}

Elizabeth A. Kensinger, Department of Psychology, Boston College, McGuinn 510, 140 Commonwealth Ave., Chestnut Hill, MA 02467; or Jessica D. Payne, Department of Psychology, University of Notre Dame, Haggar Hall, Room I22B, Notre Dame, IN 56556

E-mail: elizabeth.kensinger@bc.edu or jpayne7@nd.edu 
in the interior portion of the brain at the level of the ears. The amygdala has strong connections with a region that sits just behind it: the hippocampus, a seahorse-shaped structure that is critical for the consolidation of episodic memories (reviewed by Moscovitch \& Nadel, 1998). Because of the strong connections between these regions, amygdala activation by stress hormones during or after an emotional experience increases activity in the hippocampus, thereby facilitating memory consolidation, particularly for emotional events (McGaugh, 2004).

Neuroimaging studies demonstrate coactivation of the amygdala and hippocampus during emotional experiences and demonstrate that the simultaneous activity of these two regions increases the likelihood that an emotional experience will be remembered (reviewed by Strange \& Dolan, 2006). A difficulty with neuroimaging studies, however, is that they cannot isolate activity during consolidation, because it occurs over many hours, days, and months, and thus cannot be captured in a single snapshot of neural activity. Therefore, the correlations between amygdala and hippocampal activity could reflect effects during the initial encoding phase of memory rather than during the later consolidation phase.

Stronger evidence to pinpoint an influence of emotion - and of amygdala activation more specifically - in memory consolidation comes from studies examining how emotional memories evolve over time. While the initial effects of emotional arousal on memory can be small or even nonexistent, the effects of emotional arousal sometimes become more apparent when memory is tested after longer delays (see Kensinger, 2009, for review). In patients with damage to the amygdala, however, these effects of time do not emerge, and patients show no exaggerated effect of emotional arousal on memories assessed after a long delay as compared to a short one (LaBar \& Phelps, 1998). These findings suggest that interactions between the amygdala and hippocampus influence how emotional memories develop over time. However, none of these studies can unambiguously isolate the consolidation phase; it is always possible that patients encode or retrieve emotional information differently than healthy individuals, or that the compounding effects of amygdala activation on encoding, consolidation, and retrieval lead to these effects. Sleep provides a means of manipulating consolidation-related processes that avoids such difficulties.

\section{Sleep's Role in Emotional Memory Consolidation}

Converging evidence from many levels of analysis suggests that the sleeping brain provides ideal conditions for memory consolidation. For example, activation patterns seen during daytime task training are reactivated during subsequent sleep in both rats and humans (reviewed by O'Neill, PleydellBouverie, Dupret, \& Csicsvari, in press), and such reactivations can lead to performance enhancements the following day (Peigneux et al., 2004). Moreover, intensive training on memory tasks can alter post-training sleep architecture (see Payne, Ellenbogen, Walker, \& Stickgold, 2008, for review). The REM phase of sleep may be particularly important for modulatory effects on emotional memory (Wagner, Gais, \& Born, 2001). In humans, medial temporal regions, including the amygdala and hippocampus, are more active during REM sleep than during wakefulness, and several studies have correlated features of REM sleep with memory for emotional information (see Walker, 2009, for review). REM-rich sleep is also associated with marked increases in cortisol, a stress hormone important for the modulation of emotional memory via interactions between the amygdala and the hippocampus (Payne \& Nadel, 2004). Together, these findings suggest that although sleep is a state of behavioral quiescence, it is associated with intense neuronal activity and functional increases in brain regions necessary for emotion and memory processing.

Given this evidence, a growing number of studies have utilized sleep manipulations to examine memory consolidation. These studies overwhelmingly support a role for sleep in the long-term storage of episodic information. Many demonstrate that sleep benefits memory for neutral episodic memory materials across various modalities (see Payne, Ellenbogen, et al., 2008, for review). Interestingly, however, when emotional (especially emotionally negative) items are intermixed with neutral items at study, sleep disproportionately benefits the consolidation of emotional relative to neutral memories. For example, Wagner et al. (2001) reported that memory for negatively arousing relative to neutral narratives was facilitated after 3 hours of late-night, REM-rich sleep known to activate the hippocampus and amygdala, but not following equivalent amounts of time spent awake or in early-night, slow-waverich sleep. Similarly, others have demonstrated that a full night of sleep preferentially improves memory accuracy for recognition of negatively arousing pictures relevant to an equivalent period of daytime wakefulness (reviewed in Walker, 2009). These findings strongly suggest a role for sleep in the processing of memory for emotional experiences. More broadly, they provide compelling evidence for the traditional view of memory consolidation, as a process that solidifies memories in a veridical manner, true to their form at initial encoding (see Payne, Ellenbogen, et al., 2008, for review).

\section{Sleep Transforms Memories in Useful and Adaptive Ways}

It has long been known that memories change with the passage of time, suggesting that the process of consolidation does not always yield exact representations of past experiences. On the face of it, this may seem strikingly maladaptive, yet such flexibility in memory representation allows the emergence of key cognitive abilities, such as generalization and inference (Ellenbogen, Hu, Payne, Titone, \& Walker, 2007), prospection (Schacter, Addis, \& Buckner, 2008), and the selective preservation of useful information extracted from a barrage of incoming stimulation and experience (Payne et al., 2009).

Consistent with these ideas, growing evidence suggests that sleep does more than simply consolidate memories in veridical form; it also transforms them in ways that render memories less 
accurate in some respects but more useful and adaptive in the long run. For example, sleep leads to flexible restructuring of memory traces so that insights can be made (Wagner, Gais, Haider, Verleger, \& Born, 2004); inferences can be drawn (Ellenbogen et al., 2007); integration and abstraction can occur (Payne et al., 2009); and, importantly for our purposes, emotionally salient aspects of information can be selectively remembered over less salient, neutral aspects (Payne, Swanberg, Stickgold, \& Kensinger, 2008). In each of these cases, sleep confers a flexibility to memory that may be at times more advantageous than a literal representation of experience.

\section{Sleep Promotes Qualitative Changes in Emotional Memories}

Because emotional experiences are often complex, Payne, Swanberg, et al. (2008) examined how the different components of negative arousing memories change across periods of sleep versus wakefulness. It was unclear how the different components of a complex experience would be processed and stored in memory - whether they would change over time or remain the same, and whether periods of sleep would affect their consolidation differently than periods spent awake. Emotional scenes could be stored as intact units, suffering some forgetting over time but retaining the same relative vividness for all components. Alternatively, the components of an experience could undergo differential memory processing, perhaps with a selective emphasis on what is most salient and worthy of remembering.

We presented participants with scenes depicting negative or neutral objects against neutral backgrounds at 9 a.m. or 9 p.m. (see Fig. 1 for example stimuli). Twelve hours later, after a day spent awake or a night including at least 6 hours of sleep, we tested memory for objects and backgrounds separately, to examine how these individual components of emotional memories change across periods of sleep and wake (Fig. 2). Daytime wakefulness led to forgetting of negative arousing scenes in their entirety, with both objects and backgrounds suffering forgetting at similar rates. Sleep, however, led to a selective preservation of negative objects but not their accompanying backgrounds, suggesting that the two components undergo differential processing during sleep. This finding suggests that, rather than preserving intact representations of scenes, the sleeping brain effectively "unbinds" scenes to consolidate only their most emotionally salient, and perhaps adaptive, emotional element (see Payne et al., 2009, and Wagner et al., 2004, for additional examples of unbinding during sleep).

To remember an event accurately, one must bind its various features together and maintain these intact representations in memory (Mather, 2007). However, sleep does not always consolidate intact representations of experience, instead allowing the individual components of a memory to be flexibly recombined or selectively enhanced at the expense of less important features (e.g., Payne et al., 2009; Payne, Swanberg, et al., 2008; Wagner et al., 2004). Unbinding the individual features of a memory is essential for these processes to occur. Determining
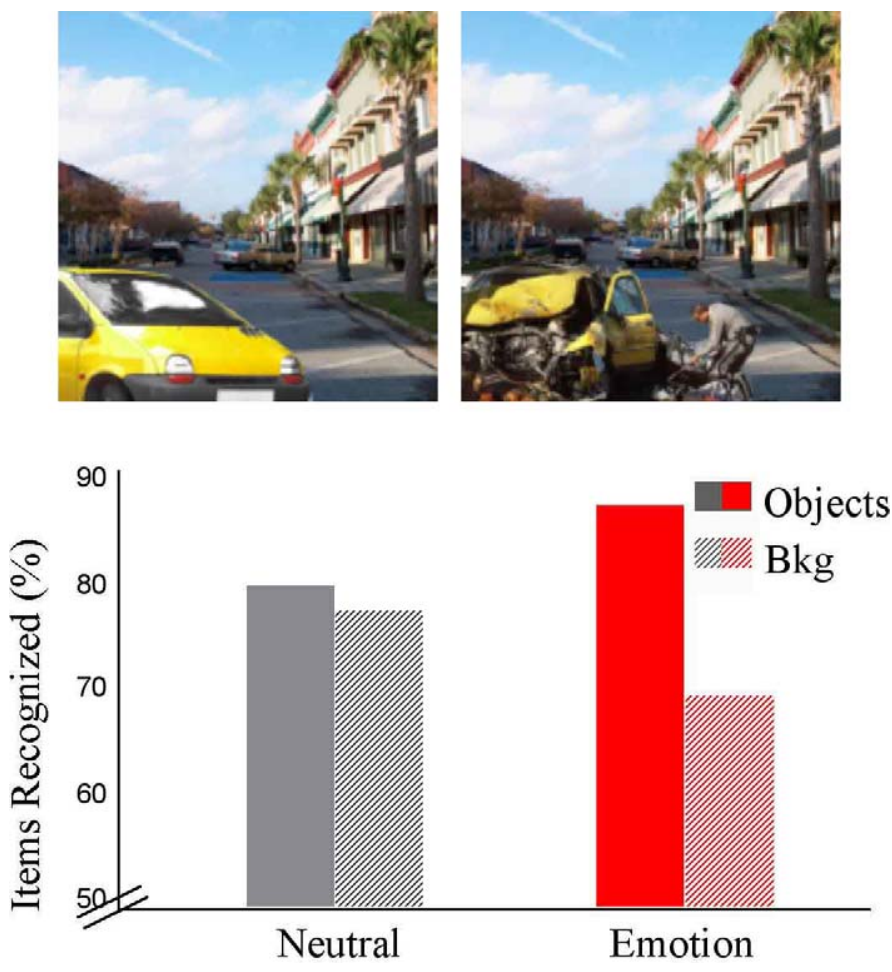

Fig. I. Sample experimental stimuli (top) and graphical representation of the trade-off effect as percentage of neutral versus emotionally arousing objects and backgrounds recognized (bottom). Stimuli with the identical neutral backgrounds and either a neutral (top left) or negatively arousing (top right) object in the foreground were presented; as shown in the graph, participants show increased recognition of emotionally arousing foreground objects with impaired recognition of neutral backgrounds (bkg).

when and under what conditions sleep preserves memories in veridical form as opposed to restructuring them for "creative cognition" has become a key question in the field (Payne et al., 2009).

We have since replicated the selective consolidation of emotional components of scene memories in two important follow-up experiments. First, we showed the same selective consolidation of emotional objects in an experiment comparing a 90-minute daytime nap to two different wake control conditions that strictly controlled for circadian and interference influences (Payne, Stickgold, Wamsley, Gibler, \& Kensinger, 2010), suggesting that at least the beginnings of memory consolidation occur after a brief amount of sleep. Moreover, we have shown that the effect is further intensified following a 24-hour and even a several-month delay, but only when sleep directly follows encoding of the emotional scenes. Across these longer delays, emotional object memories continue to be protected (i.e., they do not deteriorate further), but there is additional, and perhaps active, suppression of memory for associated backgrounds. While further work is needed to determine the precise time course of memory consolidation, these data suggest that additional nights of sleep might continue to sculpt the memory trace. 


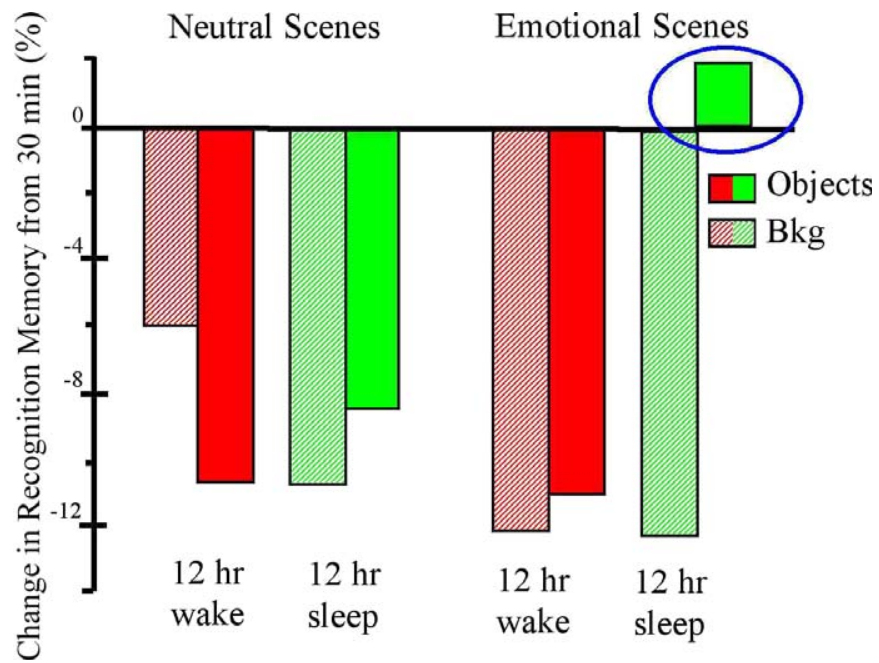

Fig. 2. Change in recognition memory for neutral and emotional objects and backgrounds after periods of waking versus sleep. Recognition memory for both central objects and neutral backgrounds (bkg) decreased from 30 minutes post-training by $6 \%$ to $12 \%$ across either 12 hours of daytime wakefulness ( 9 a.m. to 9 p.m.) or 12 nighttime hours ( 9 p.m. to 9 a.m.) including a full night of sleep ( $\geq 6$ hours of documented sleep time within this time window), except for emotional objects, memory of which improved by $2 \%$ over a night of sleep (circled green bar, right).

Finally, it is worth mentioning that in our studies, sleep exerts its strongest effect on general or "gist" memory, rather than memory for specific details. This is consistent with the findings of $\mathrm{Hu}$, Stylos-Allen, and Walker (2006), who also found that sleep benefited recognition memory for negatively arousing pictures, but only for "know" (not "remember") responses. Together, these findings suggest that, at least in some cases, sleep promotes memory for gist over memory for detail (see also Payne et al., 2009).

Collectively, these results suggest that sleep-mediated consolidation processes solidify the negative emotional aspects of an experience into a durable memory trace, while allowing the less emotional aspects to deteriorate. This finding may help us understand why memory for central, emotional information is often remembered at the expense of background details (see Fig. 1; Reisberg \& Heuer, 2004). A real-world example of such an emotional memory "trade-off" is the weapon-focus effect: Crime victims vividly remember an assailant's weapon but have little memory for other important aspects of the scene. Often these effects have been described in terms of attentional narrowing at encoding. Indeed, having a visually evocative "attention magnet" in a scene can increase the likelihood that such trade-offs occur (Reisberg \& Heuer, 2004), and neuroimaging studies have implicated the engagement of affectiveattentional processes during encoding in the formation of a memory that will only include a subset of the details processed during an event (Kensinger, 2009). Our recent sleep studies, however, emphasize that the details of emotional experiences continue to be altered with the passage of time and are fundamentally influenced by sleep-based consolidation processes.

\section{Evidence From Neuroimaging}

Investigations of the neural mechanisms of emotional-memory consolidation are further elucidating how emotional memories change over time and are ultimately stored in the brain. A recent functional magnetic resonance imaging (fMRI) study provided evidence that a single night of sleep is sufficient to provoke changes in the emotional-memory circuitry, leading to increased activity within the amygdala and the ventromedial prefrontal cortex and resulting in strengthened connectivity between the amygdala and both the hippocampus and the ventromedial prefrontal cortex (Payne \& Kensinger, in press). These findings are consistent with a study by Sterpenich et al. (2009) and suggest that sleep strengthens the modulatory effect of the amygdala on other regions of the emotional memory network, consistent with consolidation theory (McGaugh, 2004). Because amygdala engagement has been connected to selective effects on memory, this result may highlight the mechanism by which sleep intensifies the selective nature of an emotional memory: By enhancing amygdala modulation of the emotional memory network, sleep may increase the likelihood that only emotion-relevant aspects of an experience are bound into a lasting memory representation.

\section{Future Directions}

Investigating how memory trade-offs develop and change during the consolidation process is a particularly worthwhile pursuit, because trade-off effects capture many of the hallmark features of emotional memory, with emotion enhancing some - but not all — aspects of experience (Kensinger, 2009; Reisberg \& Heuer, 2004). They also provide a unique means of examining a new and vital question in sleep and memory research, which is how sleep-dependent consolidation processes preferentially select some information about an experience for long-term storage while allowing, and perhaps actively triggering, other information in memory to deteriorate. How the sleeping brain makes such calculations is currently one of the most mystifying and exciting questions in the field. The specificity of the memory effects reviewed here suggests a nuanced and selective role for sleep, and so answering this question may lead to new conceptualizations of the role that sleep plays in human cognition.

The findings reviewed here suggest that sleep-based consolidation processes are essential for the maintenance of emotional information over the long term and play a critical role in selectively preserving emotional aspects of complex experiences. In most situations, this selectivity is likely adaptive. It may be safest to home in on the emotional facets of our experience, flexibly preserving them in memory for adaptive responding to future emotional situations. If memory functions to predict and prepare for the future as much as to recall the past (Schacter et al., 2008), then selective consolidation effects make good evolutionary sense.

Nevertheless, it is certainly possible to have too much of a good thing. In affective disorders such as depression and 
PTSD, the same memory biases, perhaps in exaggerated form, might perpetuate or produce an unhealthy emphasis on the negative. Interestingly, both disorders are associated with a tradeoff known as the selective negative memory bias (e.g., Mogg, Mathews, \& Weinman, 1987). This tendency to remember the negative at the exclusion of other information is thought to contribute to the etiology and maintenance of affective disorders. Interestingly, such disorders are also associated with marked changes in REM sleep physiology, including excessive REM sleep and shortened REM sleep latency (Cartwright, 1983). Cortisol changes are also observed in depression and anxiety disorders (Payne, Nadel, Britton, \& Jacobs, 2004), which may interact with the alterations in REM sleep and emotional memory processing during sleep. Understanding the cognitive and neural mechanisms of the development and long-term consolidation of these emotional memory trade-offs, and how sleep is involved, is essential for knowledge of healthy emotional processing as well as its dysfunction. For these reasons, clarifying how, why, and under what conditions emotional memories last, and how they change over time, are critical tasks for both cognitive neuroscience and clinical psychology.

\section{Recommended Reading}

Bartlett, F.C. (1932). Remembering: A study in experimental and social psychology. Cambridge, England: Cambridge University Press. A landmark work on memory transformation, reviewing evidence that memory is not a literal reproduction of experience but rather a reconstruction of it.

Cahill, L., \& McGaugh, J.L. (1998). Mechanisms of emotional arousal and lasting declarative memory. Trends in Neurosciences, 21, 294 299. A seminal review discussing the effects of emotional arousal on memory performance.

Marshall, L., Helgadottir, H., Molle, M., \& Born, J. (2006). Boosting slow oscillations during sleep potentiates memory. Nature, 444, 610-613. Some of the strongest evidence for sleep-based memory consolidation to date.

Payne, J.D., Jackson, E.D., Hoscheidt, S., Ryan, L., Jacobs, W.J., \& Nadel, L. (2007). Stress administered prior to encoding impairs neutral but enhances emotional long-term episodic memories. Learning and Memory, 14, 861-868. Reveals that stress and elevated cortisol enhance memory for emotional events but impair memory for closely matched neutral events.

Walker, M.P., \& Stickgold, R. (2006). Sleep, memory, and plasticity. Annual Review of Psychology, 57, 139-166. A comprehensive review of the effects of sleep on memory.

\section{Declaration of Conflicting Interests}

The authors declared that they had no conflicts of interest with respect to their authorship or the publication of this article.

\section{Funding}

Preparation of this manuscript was assisted by funding from the National Science Foundation and the National Institutes of Health (MH080833).

\section{References}

Cartwright, R.D. (1983). Rapid eye movement sleep characteristics during and after mood-disturbing events. Archives of General Psychiatry, 40, 97-201.

Ellenbogen, J.M., Hu, P.T., Payne, J.D., Titone, D., \& Walker, M.P. (2007). Human relational memory requires time and sleep. Proceedings of the National Academy of Sciences, USA, 104, 7723-7728.

Hu, P., Stylos-Allan, M., \& Walker, M. (2006). Sleep facilitates consolidation of emotional declarative memory. Psychological Science, 17, 891-898.

Kensinger, E.A. (2009). Remembering the details: Effects of emotion. Emotion Review, 1, 99-113.

LaBar, K.S., \& Phelps, E.A. (1998). Arousal-mediated memory consolidation: Role of the medial temporal lobe in humans. Psychological Science, 9, 490-493.

Mather, M. (2007). Emotional arousal and memory binding. Perspectives on Psychological Science, 2, 33-52.

McGaugh, J.L. (2004). The amygdala modulates the consolidation of memories of emotionally arousing experiences. Annual Review of Neuroscience, 27, 1-28.

Mogg, K., Mathews, A., \& Weinman, J. (1987). Memory bias in clinical anxiety. Journal of Abnormal Psychology, 96, 94-98.

Moscovitch, M., \& Nadel, L. (1998). Consolidation and the hippocampal complex revisited: In defense of the multiple-trace model. Current Opinion in Neurobiology, 8, 297-300.

O’Neill, J., Pleydell-Bouverie, B., Dupret, D., \& Csicsvari, J. (in press). Play it again: Reactivation of waking experience and memory. Trends in Neurosciences.

Payne, J.D., Ellenbogen, J.M., Walker, M.P., \& Stickgold, R. (2008). The role of sleep in memory consolidation. In H. Roediger (Ed.), Learning and memory: A comprehensive reference (Vol. 2, pp. 663-685). Oxford, England: Elsevier.

Payne, J.D., \& Kensinger, E.A. (in press). Sleep leads to qualitative changes in the emotional memory trace: Evidence from fMRI. Journal of Cognitive Neuroscience.

Payne, J.D., \& Nadel, L. (2004). Sleep, dreams and memory consolidation: The role of the stress hormone cortisol. Learning and Memory, 11, 671-678.

Payne, J.D., Nadel, L., Britton, W.B., \& Jacobs, W.J. (2004). The biopsychology of trauma and memory. In D. Reisberg \& P. Hertel (Eds.), Emotion and memory (pp. 76-128). Oxford, England: Oxford University Press.

Payne, J.D., Schacter, D.L., Propper, R.E., Huang, L.W., Wamsley, E.J., Tucker, M.A., et al. (2009). The role of sleep in false memory formation. Neurobiology of Learning and Memory, 92, 327-334.

Payne, J.D., Stickgold, R., Wamsley, E., Gibler, K., \& Kensinger, E.A. (2010). A brief daytime nap selectively consolidates memory for emotional aspects of scenes. Manuscript submitted for publication.

Payne, J.D., Swanberg, K., Stickgold, R., \& Kensinger, E.A. (2008). Sleep preferentially enhances memory for emotional components of scenes. Psychological Science, 19, 781-788.

Peigneux, P., Laureys, S., Fuchs, S., Collette, F., Perrin, F., Reggers, J., et al. (2004). Are spatial memories strengthened in the human hippocampus during slow wave sleep? Neuron, 44, $535-545$. 
Reisberg, D., \& Heuer, F. (2004). Remembering emotional events. In D. Reisberg \& P. Hertel (Eds.), Memory and emotion (pp. 3-41). New York, NY: Oxford University Press.

Schacter, D.L., Addis, D.R., \& Buckner, R.L. (2008). Episodic simulation of future events: Concepts, data, and applications. Annals of the New York Academy of Sciences, 1124, 39-60.

Sterpenich, V., Albouy, G., Darsaud, A., Schmidt, C., Vandewalle, G., $\mathrm{Vu}$, T.T.D., et al. (2009). Sleep promotes the neural reorganization of remote emotional memory. Journal of Neuroscience, 29, 51435152 .
Strange, B.A., \& Dolan, R.J. (2006). Anterior medial temporal lobe in human cognition: Memory for fear and the unexpected. Cognitive Neuropsychiatry, 11, 198-218.

Wagner, U., Gais, S., \& Born, J. (2001). Emotional memory formation is enhanced across sleep intervals with high amounts of rapid eye movement sleep. Learning and Memory, 8, 112-119.

Wagner, U., Gais, S., Haider, H., \& Verleger, R., \& Born, J. (2004). Sleep inspires insight. Nature, 427, 352-355.

Walker, M.P. (2009). Overnight therapy? The role of sleep in emotional brain processing. Psychological Bulletin, 135, 731-748. 